\title{
A non-inferiority study of non-mental healthcare utilisation outcomes from care models for mental health patients with specific coexisting chronic diseases
}

Dr Michelle Jessica Pereira ${ }^{1}$, Dr Joseph D. Molina ${ }^{1}$, Dr Yap Chun Wei ${ }^{1}$, Dr Alvin Lum², Ms Ng Bee Lan², Prof Chua Hong Choon ${ }^{2}$

INSTITUTE
ofMENTAL
HEALTH
National Healthcare Group

1 Health Services and Outcomes Research, National Healthcare Group, Singapore

2 Institute of Mental Health, National Healthcare Group, Singapore

Correspondence: Michelle_Jessica_Pereira@nhg.com.sg

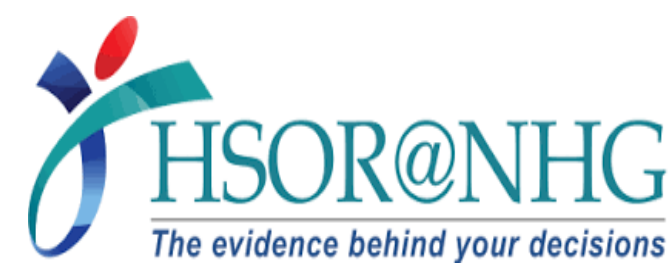

\section{BACKGROUND}

Mentally ill patients with non-mental comorbidities have higher morbidity and mortality, but poorer healthcare access. In Singapore, $42.6 \%$ of mentally ill patients have a non-mental comorbidity(Ref 1$)$. As the evidence for multimorbid care models in mental health is limited, this study compared non-mental healthcare utilisation and associated costs of different care models for patients in Singapore with coexisting psychiatric and specific non-mental chronic diseases (diabetes, hypertension or dyslipidaemia - DHL).

\section{METHODS}

A retrospective cohort study compared 3 groups of patients with a non-inferiority design, using routine administrative data (Figure 1).

1. General practice (GP) group - Patients who ever visited the GP clinic sited at the Institute of Mental Health (IMH). This GP clinic was established in 2016 to cater for IMH patients who default $\mathrm{DHL}$ care for a variety of reasons, in response to the public health challenge of multimorbidity amongst mentally ill patients. These GPs have graduate training in mental health.

2. National healthcare group polyclinics (NHGP) group - Patients who visited any NHGP, for any chronic medical condition, two or more consecutive times post index visit. Before the IMH GP clinic was in service, IMH patients were directed here for non-mental healthcare needs.

3. Psychiatrist-only group - Patients who made two or more consecutive psychiatrist outpatient consultations after their index visit, and are not GP or NHGP group patients.
Figure 1. Study design and timeframe.

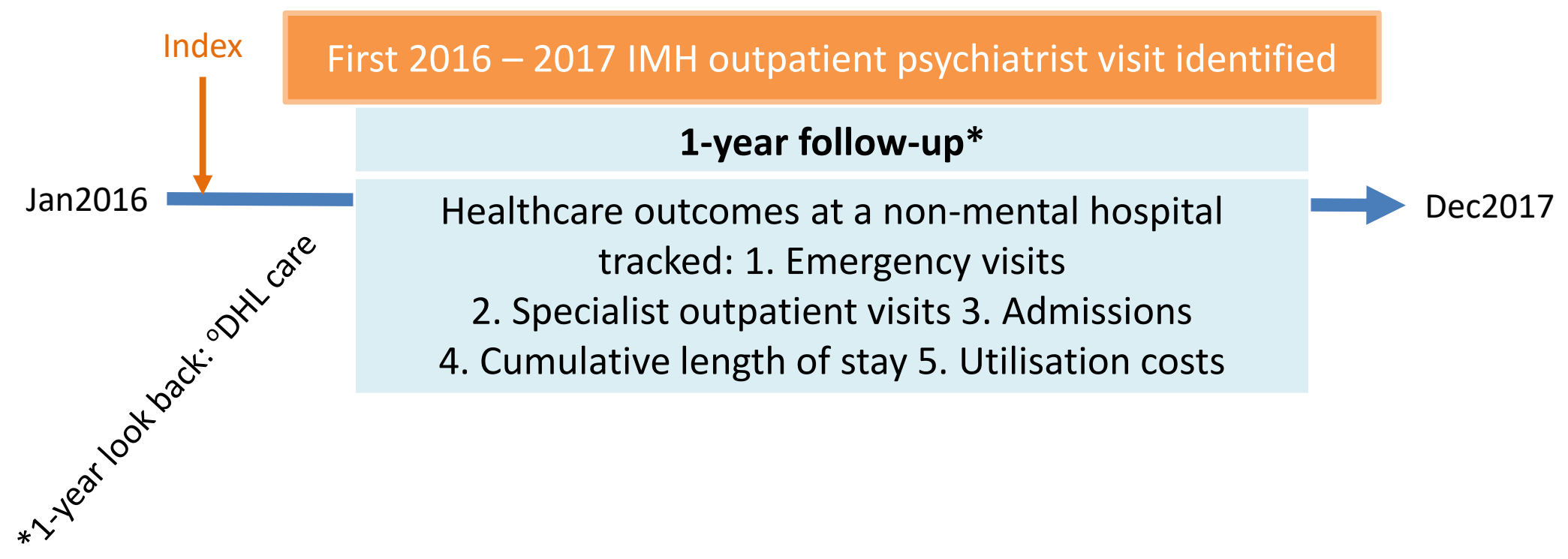

\section{ANALYSIS}

Covariates like age, gender, ethnicity, financial assistance status, and diagnosis of 7 psychiatric conditions (anxiety, depression, schizophrenia, dementia, alcohol addiction, substance addiction, bipolar disorder), DHL, and other additional non-mental chronic medical conditions that were available within the administrative database used, were extracted. These were adjusted for in the statistical analyses. Zero-inflated poisson regressions were used to analyse utilisation outcomes, and generalised linear models (gamma family, log link) were employed for costs.

\section{RESULTS}

Data from 493 patients was analysed (Figure 2). Similar emergency and specialist outpatient visits, admissions and cumulative length of stay were found between-groups (Figure 3). GP costs were similar to other groups. Considering an unknown non-inferiority equivalence margin, NHGP patients appeared to incur higher non-mental utilisation costs compared to psychiatric-only care $(\mathrm{GP}=\$ 818 ; 95 \% \mathrm{Cl}=151-1484$; NHGP $=\$ 1229 ; 95 \% \mathrm{Cl}=690-1769 ;$ psychiatrist-only $=\$ 303 ; 95 \% \mathrm{Cl}=105-501)$.

Figure 2. Patient groups.

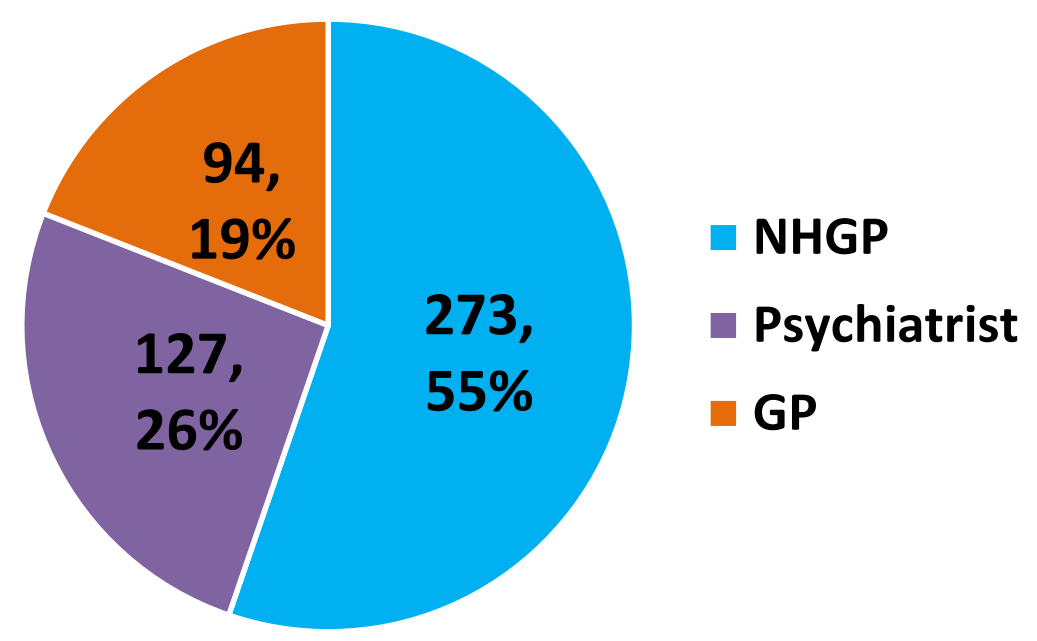

\section{CONCLUSIONS}

These care models for mental health patients with specific comorbidities demonstrated potential non-mental healthcare utilisation equivalence and some differences in associated costs. Further evaluation of cost-effectiveness is urgently needed to promptly facilitate the implementation of cost-effective multimorbid care models to improve healthcare access for this vulnerable population.

Figure 3. Non-mental healthcare utilisation comparison of mean predicted utilisation events for an average patient $(95 \% \mathrm{Cls})$ across groups.

Emergency visits

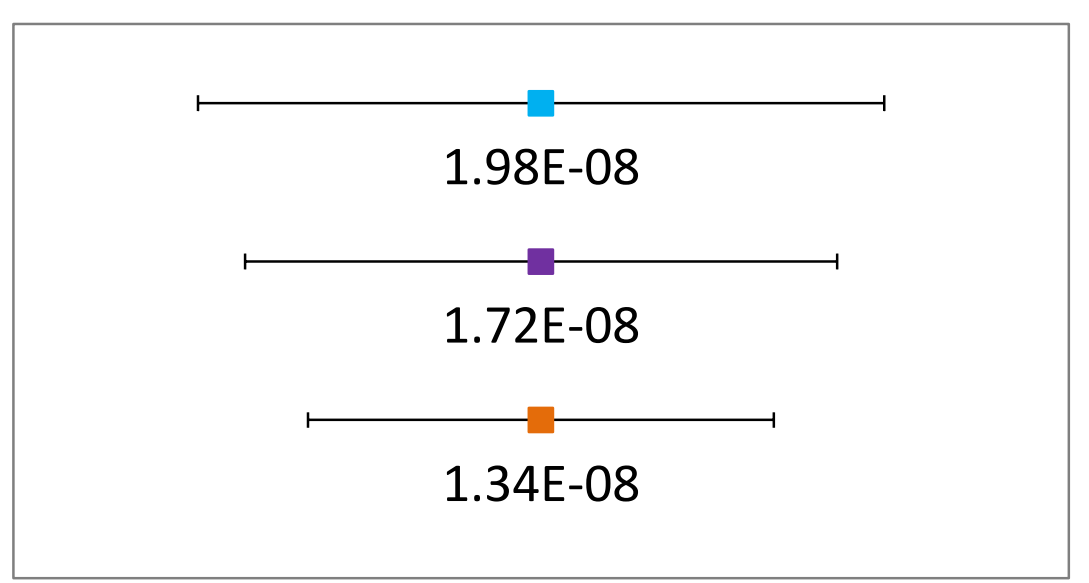

Admissions

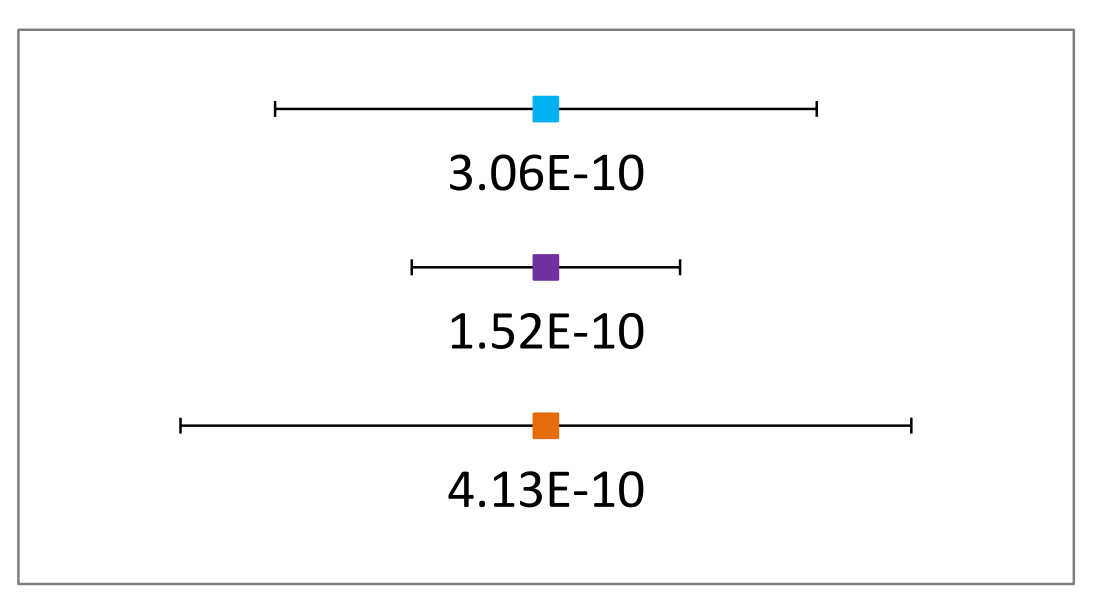

Specialist visits

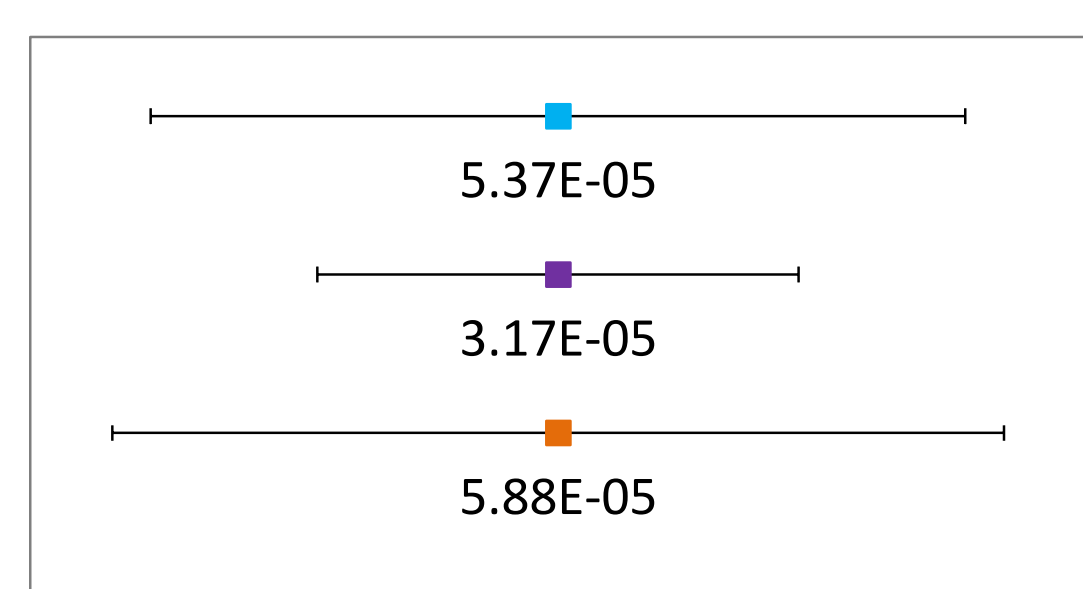

Cumulative Length of Stay

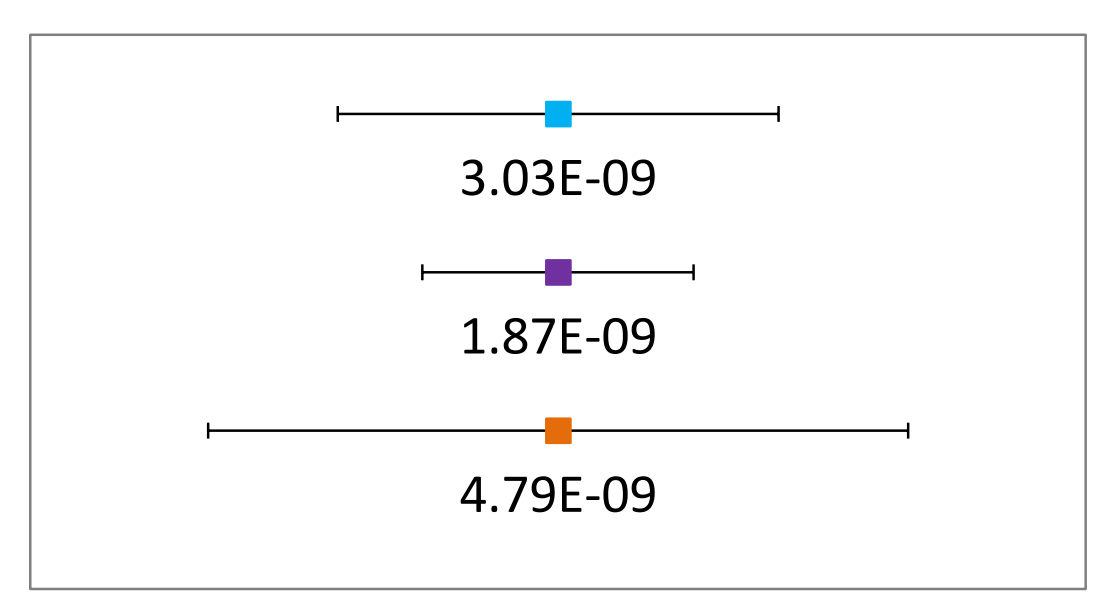

